\title{
A rare diagnosis of a focal liver lesion
}

\author{
Alberto Del Prato, ${ }^{1}$ Michela Perinetti, ${ }^{1}$ Gerardo Dessì, ${ }^{2}$ Fabio Chiesa, ${ }^{3}$ Ilan Rosenberg, ${ }^{3}$ Teseo Stefanini, ${ }^{2}$ \\ Franco Fedeli, ${ }^{2}$ Giovanni Berisso, ${ }^{3}$ Alberto Tagliafico, ${ }^{1}$ Alessandro Villa ${ }^{3}$ \\ ${ }^{1}$ Radiology Department, IRCCS AOU San Martino-IST, University of Genova, Genova; ${ }^{2}$ Ospedale Sant'Andrea, La Spezia; \\ ${ }^{3}$ Ospedale San Bartolomeo, Sarzana (SP), Italy
}

\begin{abstract}
Splenosis can be considered as a benign condition due to the presence of heterotopic splenic tissue in abdomen, thorax and pelvis because of a massive splenic trauma or surgery. Here we report the case of a patient with an unknown hepatic mass, chronic hepatitis $\mathrm{C}$, liver cirrhosis and a previous splenectomy after abdominal trauma. In our case lesion could not be clearly defined by ultrasound, computed tomography and magnetic resonance. Classical features of malignancy were not demonstrated at computed tomography, while at magnetic resonance imaging the differential diagnosis of the lesion appeared quite difficult and debate. Then an ultrasound-guided biopsy demonstrated the liver mass in left hepatic lobe consisted of splenic tissue and some millimetric accessory spleens in the left upper quadrant. So the possibility of an intra-hepatic splenosis should be taken into account in patients with an unknown liver mass and a history of previous abdominal trauma, followed by splenectomy. The conclusive diagnosis of intra-hepatic splenosis was given by ultrasound-guided biopsy.
\end{abstract}

\section{Introduction}

Liver masses in patients with chronic hepatitis $\mathrm{C}$ $(\mathrm{HCV})$ and liver cirrhosis raise the suspicion of a nodule of hepatocellular carcinoma (HCC). The detection of certain benign or malign features cannot be so easy in this situation.

A clear interpretation of diagnostic imaging, based on ultrasound (US), computed tomography (CT) and magnetic resonance imaging (MRI) was not possible, because imaging findings were quite conflicting.

We report a case of intra-hepatic splenosis in a patient with hepatitis $\mathrm{C}$, liver cirrhosis, a history of al-

Correspondence: Alberto Del Prato, via Dei Colli 95, 19121

La Spezia, Italy.

Tel.: +39.340.8988104.

E-mail: albertodelprato@alice.it

Key words: splenosis, liver cirrhosis, focal lesion, splenectomy, trauma.

Conflict of interests: the authors declare no potential conflict of interests.

Received for publication: 25 March 2014.

Revision received: 28 May 2014.

Accepted for publication: 4 June 2014.

This work is licensed under a Creative Commons Attribution NonCommercial 3.0 License (CC BY-NC 3.0).

(C) Copyright A. Del Prato et al., 2015

Licensee PAGEPress, Italy

Italian Journal of Medicine 2015; 9:71-76

doi:10.4081/itjm.2015.506 coholism and an abdominal trauma with splenic rupture, followed by splenectomy.

\section{Case Report}

A 45-year-old Caucasian man from Italy was admitted to our hospital, because he was sent by his general practitioner, who found a very high value (537 $\mathrm{mg} / \mathrm{dL}$ ) of glycemia, suspecting a diabetes mellitus, not well ruled by insulin; beyond the patient complained about polyuria and polydipsia. In his medical history it was appeared a protracted alcoholic abuse. He had no jaundice, no fever, no weight loss and at objective exam it was only possible to feel an enlarged liver. His remote pathological anamnesis included an abdominal trauma, followed by a splenectomy. In January 2013 he underwent to surgical operation because of a post-traumatic brain hematoma and in that situation, after mindless laboratory exams, it discovered the presence of a chronic HCV infection.

At admission, glycemia was $537 \mathrm{mg} / \mathrm{dL}$, serum aminotransferases were as elevated [aspartate transaminase (AST) $121 \mathrm{U} / \mathrm{L}$, alaninotransaminase (ALT) 155 $\mathrm{U} / \mathrm{L}$ with reference standard values of 2-37 UI/L both for AST and for ALT] as the cholestasis indexes, such as bilirubin $(2.2 \mathrm{mg} / \mathrm{dL})$ and $\gamma$-glutamyl transpeptidases $(251 \mathrm{U} / \mathrm{L})$; alkaline phosphatase was normal $(186 \mathrm{U} / \mathrm{L})$. $\alpha$-fetoprotein was at the superior limits $(8 \mathrm{ng} / \mathrm{mL}$ with reference standard values of $0-8 \mathrm{ng} / \mathrm{mL}$ ).

An US exam (My Lab 70 Esaote, probe $5 \mathrm{MHz}$ ) revealed the presence of an enlarged liver characterized by a fatty fibrotic pattern without positive evidence of focal liver lesions.

Then the patient underwent a CT exam (Aquilion, 
Toshiba Corp., Minato, Tokyo) with acquisition of images before and after intravenous administration of iodinated contrast agent (Visipaque 320) in arterial, portal and equilibrium phases; this exam allowed to identify, in a context of a fatty fibrotic enlarged liver, a focal solid round lesion with a diameter of $6 \mathrm{~cm}$. It was not possible to identify any finding in the unenhanced contrast phase, but the lesion was sharply hyperdense in all phases after contrast administration (Figure 1). No evidence of wash out was appreciated. Further CT exam detected, in a frame of splenectomy, some millimetric accessory spleens in the asplenic host.

So, for a better characterization, it was necessary to execute a liver MR with hepatic specific contrast agent.

Then an MR exam (1.5 T, Achieva; Philips Healthcare, Amsterdam, the Netherlands) was performed using GRE T1-weighted images in phase and in opposition phase, TSE-T2 weighted images with and without fat suppression, DWI sequences and dynamic imaging after intravenous administration of standard gadoterate $(0.025 \mathrm{mmol} / \mathrm{kg}$, Primovist, Bayer Schering Healthcare, Berlin, Germany), obtaining images also in hepato-specific phases after $15 \mathrm{~min}$; the exam revealed an enlarged fatty-fibrotic liver and, in the left liver lobe, it was confirmed the presence of the note focal nodular round lesion, having a diameter of $6 \mathrm{~cm}$. It was hyper-intense in TSE-T2 weighted images, hypo-intense in TSE T1 in phase and out of phase, on the ADC map the nodule demonstrated very low signal to refer with restricted diffusion, then it was hyper-vascular in arterial phase and it showed the phenomenon of wash out, typical of $\mathrm{HCC}$ in equilibrium phase; the lesion was hypo-intense in the hepato-biliary phase respect to the surrounding liver (Figures 2 and 3).

These MR features were referred to a focal liver lesion, compatible with HCC. But anyway the accessory spleens were also detected in the asplenic host.

So it was decided to send the patient to perform a US-guided biopsy using a 18 Gauge needle. No adverse reactions were noticed after the bioptical procedure. Histological findings appeared to be referred to splenic heterotopic intra-hepatic tissue. According to it, hepatic cells and fatty-fibrotic tissue were not found in the lesion; it was possible to only detect red blood cells, lymphoid tissue and granulocytes (Figure 4).

The result of US-guided biopsy allowed to exclude the potential malignancy of the lesion and to aim to a correct diagnosis. Anyway the suspicion of HCC was removed and, because of the benign nature of the lesion, represented by a condition of intra-hepatic splenosis, it was decided to avoid every surgical procedure and the patient was discharged; so he could

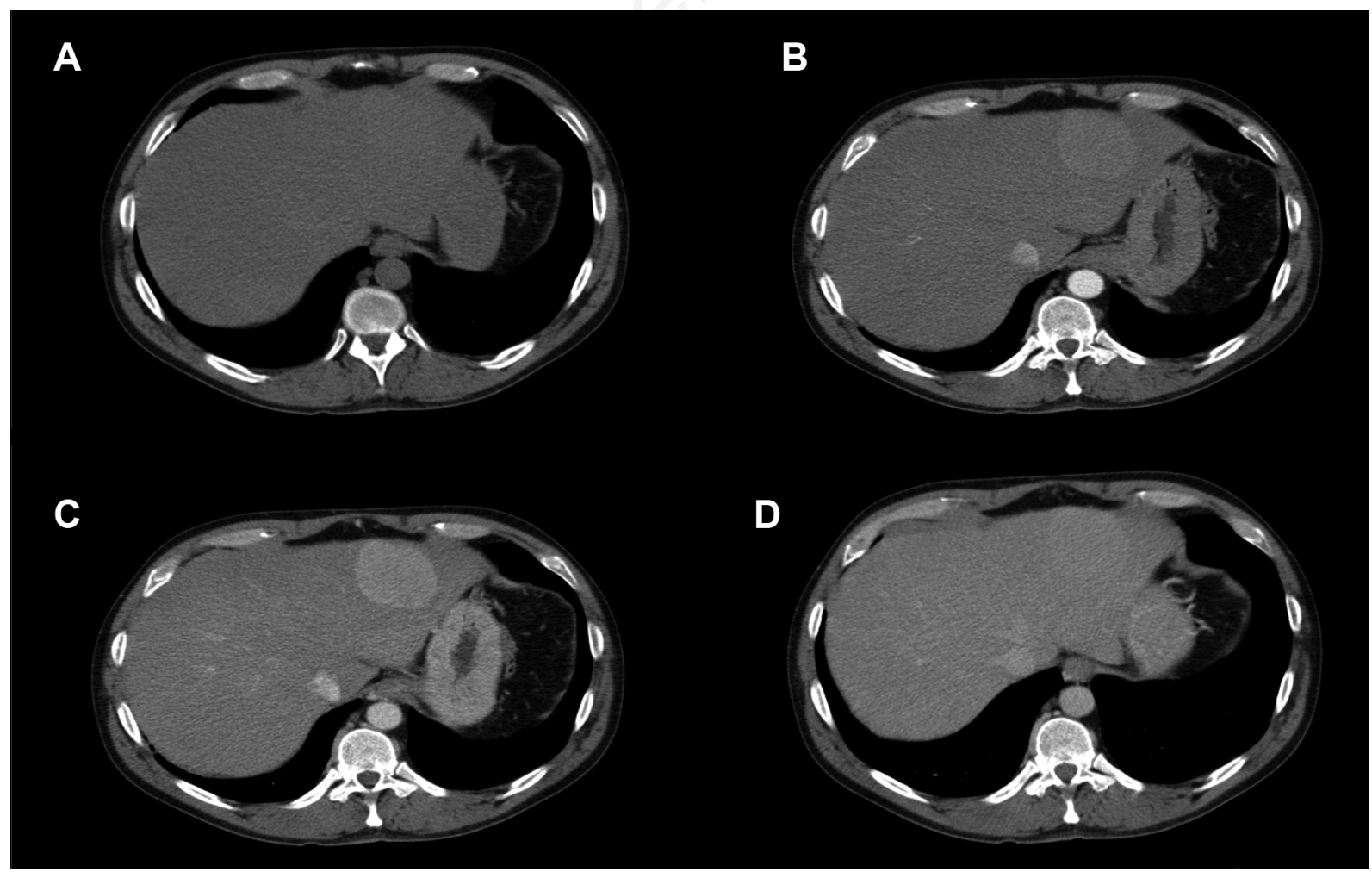

Figure 1. Computed tomography (CT) patterns of the hepatic focal round lesion. (A) An unenhanced transverse CT scan does not show the lesion. The focal round lesion appears to be hyper-dense in arterial phase CT transverse scan (B), hyper-dense in portal venous phase (C), and also hyper-dense in equilibrium phase (D). 
continue with his usual follow-up exams for checking the liver, characterized by the known fatty-fibrotic pattern, and his normal therapies.

\section{Discussion}

Splenosis is the heterotopic auto-transplantation of splenic tissue and occurs in 16 to $67 \%$ of patients after traumatic spleen rupture or spleen surgery. ${ }^{1}$ It is important to distinguish splenosis from accessory spleens. Accessory spleens are located on the left side of the mesogastrium in the region of the splenopancreatic or gastrosplenic ligaments. ${ }^{2}$ Given its innocuous nature, splenosis may be beneficial after traumatic splenic rupture. Indeed, there is no indication to remove splenosis if the patient is asymptomatic. ${ }^{3}$ The implants are usually small and multiple and hundreds of them can be localized throughout the peritoneal cavity. ${ }^{3}$ The fragments can implant anywhere where they have access, but are most commonly found on the serosal surfaces of the small intestine, greater omentum, mesentery, under the surface of diaphragm and in the pelvis. ${ }^{1}$

There have been reports of thoracic splenosis following diaphragmatic rupture and subcutaneous splenosis following gunshot wounds through the spleen.,4

Splenosis is a rare entity. To date, around 100 cases were reported in the literature until $2004 .{ }^{5}$

It is important to understand the difference between splenosis and accessory spleens. Rupture of a pathologic spleen is more likely to occur than that of a normal one, and may be either spontaneous or traumatic. ${ }^{6}$ In newborn infants splenic rupture may occur in severe hemolytic disease whereas in the older children and adolescents such rupture most commonly occurs in infectious mononucleosis. ${ }^{6}$ Traumatic rupture of the spleen may occur from a sharp blow to the left flank, as, for example, in the automobile accidents, a direct blow or striking a projecting object while running, sledding, bicycling or the like. ${ }^{6}$ Removal of the spleen offers the greatest possibility for recovery and usually no deleterious effect
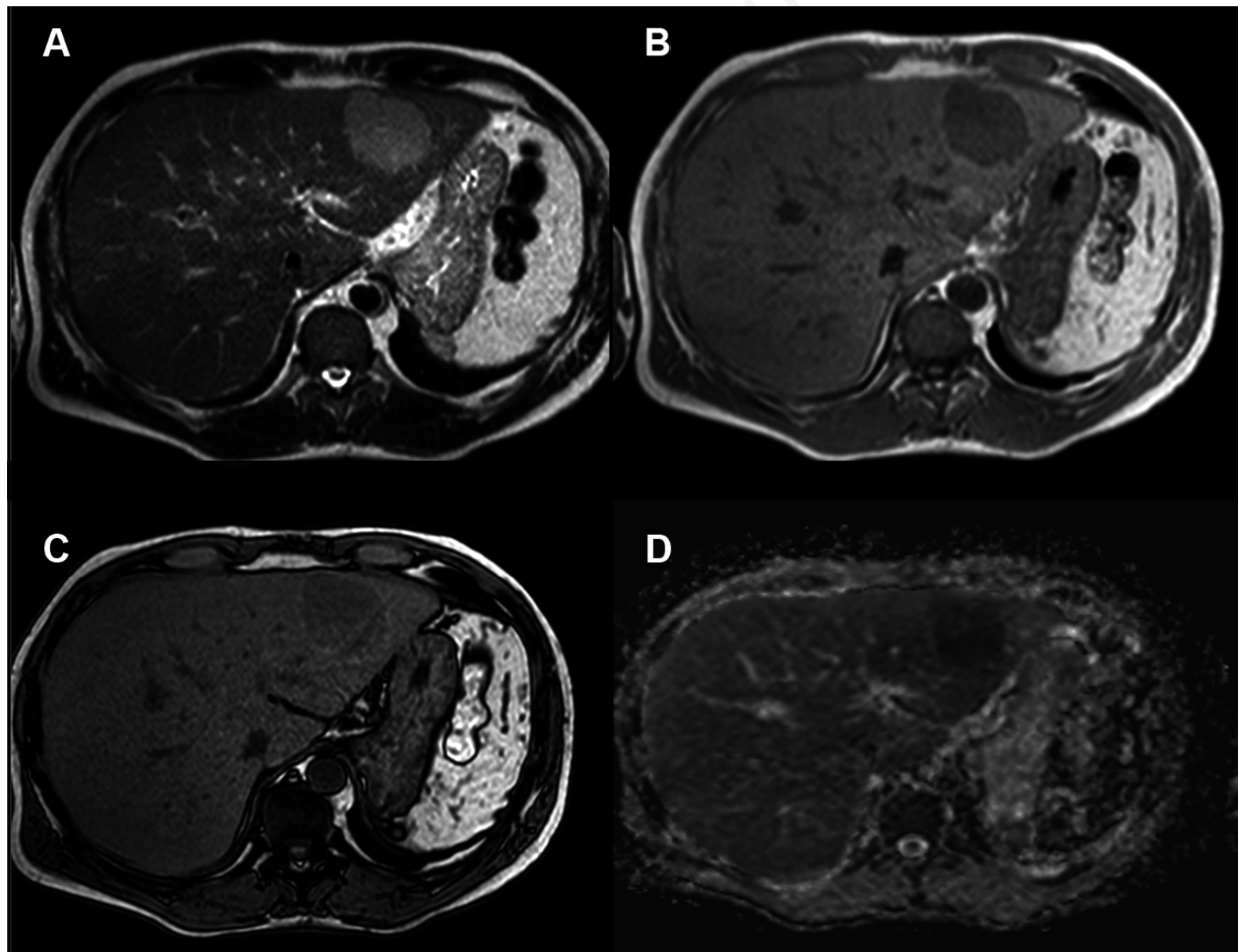

Figure 2. Magnetic resonance findings of the hepatic focal lesion. A TSE-T2 weighted image shows a hyper-intense lesion in the left hepatic lobe (A), then the nodule appears to be hypo-intense in TSE T1-weighted in phase (B) and out of phase (C) images and at the ADC map it has a highly low signal (D). 
upon the subsequent growth and development of the child is observed. ${ }^{6}$ The mechanism behind auto-transplantation initiated with the splenic rupture involves mainly seeding of damaged splenic pulp into the adjacent cavities. ${ }^{6}$ A second mechanism is the hematogenous spread of splenic pulp. A novel hypothesis that appeared in the context of hepatic splenosis is that splenic erythrocyte progenitor cells enter the liver via the portal vein, and then grow in response to tissue hypoxia. ${ }^{7}$ A definite preoperative diagnosis of abdominal splenosis requires a high index of suspicion and should be established cautiously. ${ }^{6}$ A detailed medical history concerning previous abdominal trauma as well as thorough physical examination is essential for making a preliminary diagnosis of abdominal splenosis. ${ }^{6}$ Lack of typical chronic changes in the blood count often presents after splenectomy like Howell-Jolly bodies, increase in the number of reticulocytes, sometimes also lymphocytes, monocytes, eosinophils, and thrombocytosis and protective levels of anti-pneumococcal antibodies in a non-vaccinated patient should make a gastroenterologist aware of this rare condition. ${ }^{6}$ Most of patients with a diagnosis of intra-he- patic splenosis are completely asymptomatic and an undefined mass is usually detected in a casual way. Abdominal ultrasound and radiological studies show only limited diagnostic value in abdominal splenosis. ${ }^{8}$ Sonographic findings are not specific and reveal round and oval soft-tissue masses in the various locations. ${ }^{8}$

Low density of splenic tissue makes it difficult to visualize on standard X-rays. CT reveals the number, shape, size, location but not identity of the nodules. ${ }^{8}$ Non-characteristic sonographic and radiological picture of abdominal splenosis may be confused with numerous conditions such as metastatic disease, abdominal lymphoma, carcinomatosis, hemangiomatosis, peritoneal mesothelioma, multifocal endometriosis, adenomas, primary renal or hepatic malignancy, gliomatosis peritonei, granulomatous peritonitis, accessory spleens or reactive adenopathy. ${ }^{8}$

It is important to have a clear diagram of all possible differential diagnoses in patients with hepatitis $\mathrm{C}$ and fatty-fibrotic liver, a focal liver mass and, in this context, it is essential to take into account at least the most important diseases, such as focal nodular hyper-
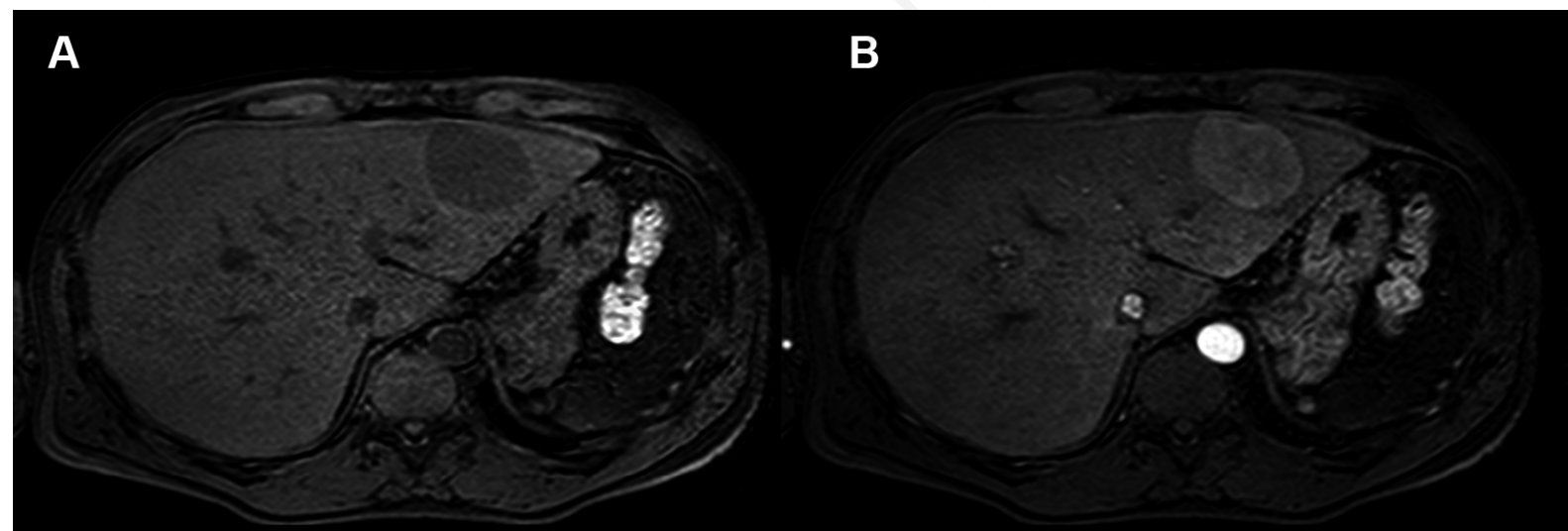

C

D

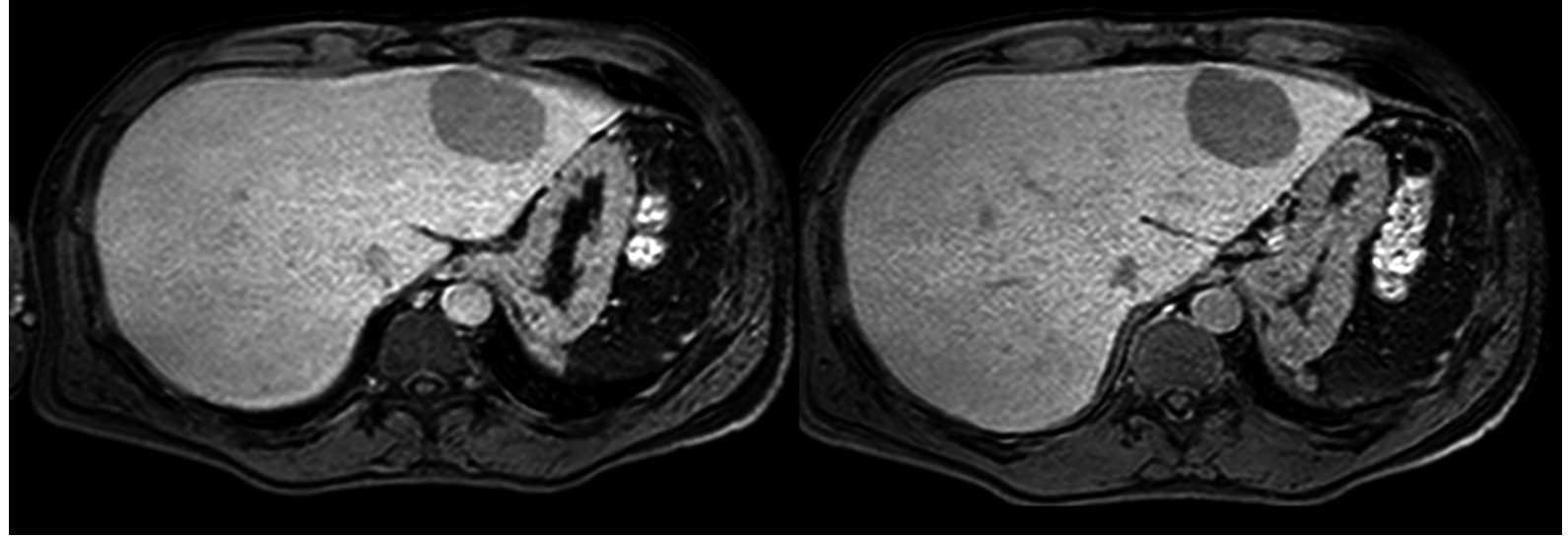

Figure 3. Magnetic resonance (MR) features of the round focal lesion. Before gadoterate administration GRE T1weighted MR image shows the round focal lesion as hypo-intense (A), then, after gadoterate intravenous administration, the lesion has a hyper-vascular pattern in arterial phase (B), it shows the phenomenon of wash out in equilibrium phase (C) and it presents a hypo-intense behavior in the hepato-biliary phase (D). 
plasia, adenoma, hemangioma, dysplastic and regenerative nodules and, of course HCC. Transmission of $\mathrm{HCV}$ and the following development of chronic hepatitis $\mathrm{C}$ used to be frequent in the past in needingtransfusion patients after an important polytrauma.

Typically, HCC is a focal liver lesion with high signal intensity on T2-weighted MR images and variable signal intensity on T1-weighted images, that shows intense enhancement during the arterial phase of dynamic gadolinium enhanced MR imaging, is usually iso-hypointense during the portal venous phase and shows washout of contrast material respect to the surrounding liver in the equilibrium phase. Using hepatospecific contrast agents, such as Gd-BOPTA (gadolinium benzyloxypropionictetraacetate) or GdDTPA (diethylenetriaminepentaacetic acid) the HCC lesion usually appears hypo-intense respect to the liver parenchyma in the delayed phase. ${ }^{9}$ In MR imaging the normal adult spleen is hypo-intense on T1-weighted imaging and hyper-intense on T2-weighted imaging with respect to the liver. Occasionally, the spleen may be hypo-intense on T2-weighted imaging because of iron deposition. The low signal intensity of the splenic tissue even after contrast administration may be due to iron deposition. ${ }^{10}$ During dynamic MRI (or CT studies) the spleen on the arterial phase demonstrates a heterogeneous patterns related to the unique anatomic structure due to variable rates of flow through the cords of red and white pulp.

On the basis of Lin et al.'s observation, the signal intensities and the enhancing patterns of the splenic implants on T1- and T2- weighted images and on dynamic MR images appear to be identical to those of a normal spleen. ${ }^{11}$ Normally splenic heterotopic implants have heterogeneous enhancement in the arterial phase of dynamic MR imaging and homogeneous enhancement in the portal and equilibrium phases. Ishibashi et al. reported that on MRI, the lesions are hypo-intense on T1weighted images and hyper-intense on T2-weighted images. After administration of superparamagnetic iron oxide (SPIO), the lesions usually show decreased signal intensity but remain slightly hyper-intense relative to liver parenchyma on T2-weighted images. ${ }^{12}$ The phagocytic reticuloendothelial cells located in the liver and spleen show a tissue-specific distribution after intravenous SPIO injection and they show the loss of signal intensity on T2-weighted MRI, while malignant tumor shows no signal loss because it does not have reticuloendothelial cells. MRI also has the advantages over nuclear medicine techniques of a higher spatial resolution. ${ }^{13}$ So, in conclusion, at standard MRI splenic implants look like normal spleen (if present), hypo-intense or hyper-intense depending on presentation (T1 and $\mathrm{T} 2$, respectively) with heterogeneous positive contrast enhancement (gadolinium-based) and MRI is not very useful in differential diagnosis. ${ }^{11}$ Recently, several pa- pers have proposed, as a novel technique for diagnosing splenosis, ferumoxides enhanced MRI after i.v. administration of superparamagnetic iron oxide particles that are removed from the circulation by the phagocytic reticuloendothelial system of the liver and spleen. ${ }^{14,15}$ This type of MRI combines a physiologic test of reticuloendothelial system uptake with the anatomic detail of MRI. ${ }^{6}$ At present, non-invasive Technetium (Tc) $99 \mathrm{~m}$ radionuclide scanning is the mainstay in the diagnosis of splenosis. Tc-99m sulphur colloid is sequestered in the reticuloendothelial system and detects heterotopic splenic tissue. ${ }^{16}$ If differentiation from hepatic tissue is necessary, a $5 \mathrm{mCi}(185 \mathrm{MBq}) \mathrm{Tc} 99 \mathrm{~m}$-tagged heatdamaged autologous red blood cells (RBCs) or Indium 111-labeled platelets scintigraphy can be performed. ${ }^{8}$ RBCs scintigraphy, although not free from the risk of adverse effects, seems to be more sensitive in early splenosis, functional hyposplenism or poor splenic uptake as well as when the spleen and the liver overlap, causing poor visualization of splenic tissue by the sulphur colloid test. If the diagnosis is confirmed preoperatively by appropriate radionuclide modalities in a patient with history of abdominal trauma, biopsy or laparotomy can be avoided. ${ }^{8}$ Because the splenic tissue may be partially or fully functioning and therefore may have some beneficial immune function for the patient, the management of this entity should be conservative. Single photon emission-CT/CT fusion imaging based on Tc-99m sulfur colloid can be used to give supplemental three-dimensional anatomical information. ${ }^{17}$

The surgical approach is recommended in cases of symptomatic or complicated splenosis and in some patients with hematological disease for whom splenectomy is beneficial and splenic auto-transplantation must be avoided (like congenital spherocytosis). ${ }^{6}$ In this subset of patients preoperative use of imaging

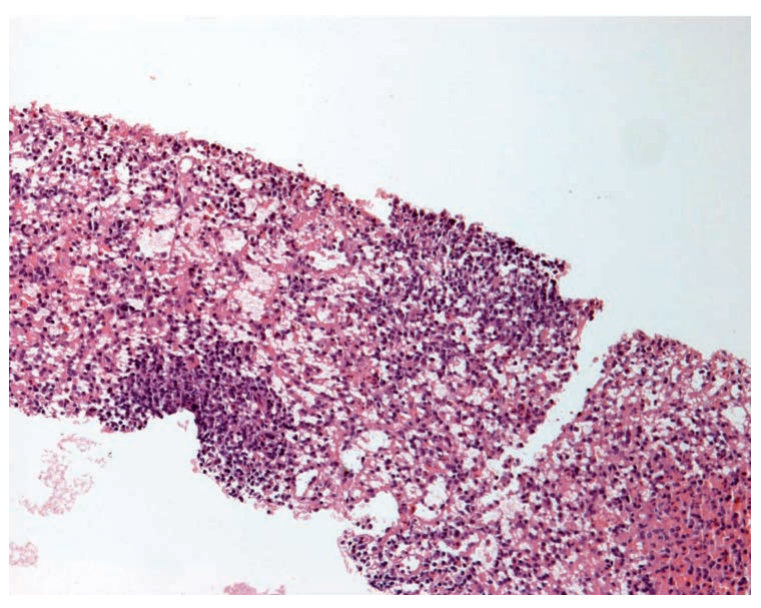

Figure 4. A representing splenic tissue image obtained by hematoxylin and eosin staining protocol (10x). 
methods might improve diagnostic certainty and contribute to a well-planned surgical intervention. The surgical approach should be also recommended in case of an uncertain diagnosis, especially when scintigraphic methods are not readily available and there is a suspicion of a malignant disease, because distinguishing the nature of the abdominal mass considerably modifies the management. ${ }^{6}$ So the current opinion is that, when the splenosis is incidentally diagnosed in an asymptomatic patient, complete surgical removal is not indicated. ${ }^{6}$

In the last years elastography, based on FibroScan, has developed, becoming a novel rapid, non-invasive and reproducible method for measuring not only liver stiffness, but also hepatic focal lesions stiffness. It is thought that in the future elastography can be used as a potential diagnostic modality in discrimination of $\mathrm{HCC}$ focal lesions from other non $\mathrm{HCC}$ focal lesions. ${ }^{18}$ Tissue stiffness is directly proportional to the square of shear wave velocity: the stiffer the tissue, the faster the shear wave propagates. ${ }^{19}$ So, theoretically, the elastography can also assess stiffness of hepatic tumors, but the diameter of the tumor should be currently between 25 and $65 \mathrm{~mm}$ from the surface. ${ }^{19}$ Furthermore the most recent studies have still many technical difficulties in the measurement of stiffness of hepatic focal lesions, which require that lesions to be large with a diameter of more than $4 \mathrm{~cm}$ and are localized in the right hepatic lobe, near the surface in patients with no ascites, excluding a great number of patients with small multiple focal hepatic lesions. ${ }^{18}$ Even though FibroScan elastography could have a role in this kind of clinical situations, in this specific setting, intra-hepatic splenosis was localized in the left liver lobe and not in the right hepatic segments, so it would not be possible to look for the optimal right intercostal position, using B-mode ultrasound.

Normally hepatocellular carcinoma focal lesions are much stiffer than lymphoma, metastasis, sarcoidosis focal lesions and, of course intra-hepatic splenosis. So it is widely considered that FibroScan may be a useful non-invasive method in the prediction of hepatocellular carcinoma in the future. ${ }^{18}$

\section{Conclusions}

Intra-hepatic splenosis represents a very rare diagnosis, which should be considered in unknown focal liver lesion presenting patients who previously had an abdominal trauma with following spleen rupture.

At MR exam with administration of gadoliniumchelates as contrast material, the lesion exhibited the same signal of HCC, because it presented hyperintensity of signal in arterial phase and rapid washout in portal venous and equilibrium phases.

Then the final diagnosis of splenosis was achieved by the US-guided biopsy that removed every doubt.

\section{References}

1. Fleming CR, Dickson ER, Harrison EG. Splenosis: autotransplantation of splenic tissue. Am J Med 1976;61: 414-9.

2. Menth M, Herrmann K, Haug A, et al. Intra-hepatic splenosis as an unexpected cause of a focal liver lesion in a patient with hepatitis $\mathrm{C}$ and liver cirrhosis: a case report. Cases J 2009;2:8335.

3. D’Angelica M, Fong Y, Blumgart LH. Isolated hepatic splenosis: first reported case. HPB Surg 1998;11:39-42.

4. Hibbeln, JF, Wilbur AC, Schreiner V, et al. Subcutaneous splenosis. Clin Nucl Med 1995;20:591-3.

5. Di Costanzo GG, Picciotto FP, Marsilia GM, et al. Hepatic Splenosis misinterpreted as hepatocellular carcinoma in cirrhotic patients referred for liver transplatation: report of two cases. Liver Transpl 2004;10:706-9.

6. Ksyadzina D, Pena AS. Abdominal splenosis. Rev Esp Enferm Dig 2011;103:421-6.

7. Kwok CM, Chen YT, Lin HT, et al. Portal vein entrance of splenic erythrocytic progenitor cells and local hypoxia of liver, two events cause intrahepatic splenosis. Med Hypotheses 2006;67:1330-2.

8. Ksyadzina D. A case report of abdominal splenosis - a practical mini-review for a gastroenterologist. J Gastrointestin Liver Dis 2011;20:321-4.

9. Hussain SM, Reinhold C, Mitchell DG. Cirrhosis and lesion characterization at MR. Imaging Radiogr 2009; 29:1637-52.

10. Siegelman ES, Mitchell DG, Semelka RC. Abdominal iron deposition: metabolism, MR findings, and clinical importance. Radiology 1996;199:13-22.

11. Lin W-C, Lee R-C, Chiang J-H, et al. MR features of abdominal splenosis. AJR Am J Roentgenol 2003;180: 493-6.

12. Ishibashi M, Tanabe Y, Miyoshi H, et al. Intrathoracic splenosis: evaluation by superparamagnetic iron oxideenhanced magnetic resonance imaging and radionuclide scintigraphy. Jpn J Radiol 2009;27:371-4.

13. Kang KC, Cho GS, Chung GA, et al. Intrahepatic splenosis mimicking liver metastasis in a patient with gastric cancer. J Gastric Cancer 2011;11:64-8.

14. Berman AJ, Zahalsky MP, Okon SA, et al. Distinguishing splenosis from renal masses using ferumoxide-enhanced magnetic resonance imaging. Urology 2003;62:748.

15. Prosch H, Oschatz E, Pertusini E, et al. Diagnosis of thoracic splenosis by ferumoxides-enhanced magnetic resonance imaging. J Thorac Imaging 2006;21:235-7.

16. Wojcik J, Grodzki T, Kozlowska I, et al. Usefulness of the scintigraphic assessment of the thoracic splenosis a case report. Pneumonol Alergol Pol 2008;76:456-9.

17. Short NJ, Hayes TG, Bhargava P. Intra-abdominal splenosis mimicking metastatic cancer. Am J Med Sci 2011;341:246-9.

18. Yousef M, Elsharkawy A, El Beshlawy M, et al. Use of ultrasonic transient elastography (FibroScan) in the assessment of hepatic focal lesion stiffness. Open J Gastroenterol 2013;3:107-12.

19. Castera L, Vergniol J, Foucher J, et al. Prospective comparison of tran-sient elastography, Fibrotest, APRI, and liver biopsy for the assessment of fibrosis in chronic hepatitis C. Gastroenterology 2005;128:343-50. 